\title{
Accurate optical reference catalogs
}

\author{
Norbert Zacharias \\ U.S. Naval Observatory (USNO), \\ 3450 Massachusetts Avenue NW, Washington, DC 20392-5420, USA \\ email:nz@usno.navy.mil
}

\begin{abstract}
Current and near future all-sky astrometric catalogs on the ICRF are reviewed with the emphasis on reference star data at optical wavelengths for user applications.
\end{abstract}

Keywords. astrometry, catalogs, surveys, reference systems, stars: kinematics, telescopes, instrumentation: detectors

The standard error of a Hipparcos Catalogue star position is now about 15 mas per coordinate. For the Tycho-2 data it is typically 20 to 100 mas, depending on magnitude.

The USNO CCD Astrograph Catalog (UCAC) observing program was completed in 2004 and reductions toward the final UCAC3 release are in progress; for updates see <ad.usno.navy.mil/ucac>. This all-sky reference catalog will have positional errors of 15 to 70 mas for stars in the 10 to 16 mag range, with a high degree of completeness, including positions of double stars from blended images. Proper motions for the about 60 million UCAC stars will be derived by combining UCAC astrometry with available early epoch data, including yet unpublished measures (StarScan) of the complete set of AGK2, Hamburg Zone astrograph and USNO Black Birch programs, reaching up to 14 th magnitude from about 1900, 2300, and 700 plates, respectively. Dedicated deep CCD imaging of about 600 QSOs obtained between 1996 and 2004 is part of the UCAC program to allow a direct tie to the ICRF independent of Hipparcos.

Other accurate optical astrometric catalogs are the 14th Carlsberg Meridian Catalog (CMC14) going deeper $(V=17)$ than UCAC and covering $-30^{\circ}$ to $+50^{\circ}$ declination, the Bordeaux zone PM2000 catalog with block-adjustment-type reductions of early (AC, Carte du Ciel) and new (scanning CCD) observations of about 2.7 million stars in the $+11^{\circ}$ to $+18^{\circ}$ zone (excellent proper motions), and the ongoing Southern Proper Motion (SPM) survey (Yale, San Juan), providing absolute proper motions in large areas of the southern sky from ties to background galaxies, similar to the already completed Northern Proper Motion (NPM, Lick Observatory) program.

Accurate positional and proper motion data are combined in the Naval Observatory Merged Astrometric Dataset (NOMAD) which includes Hipparcos, Tycho-2, UCAC2, USNO-B1, NPM + SPM plate scan data for astrometry, and is supplemented by multiband optical photometry as well as 2MASS near infrared photometry, covering all stars down to about 20th magnitude. Accurate positions of fainter stars in selected areas are available in the Sloan Digital Sky Survey (SDSS) and the Deep Astrometric Standards (DAS) project using 4-meter class telescopes on 4 fields of 10 sq.deg. size each to 24th magnitude.

The optical design of the USNO Robotic Astrometric Telescope (URAT) is complete and the world largest, monlithic CCD chip (111 million pixel, $95 \mathrm{~mm}$ square) was fabricated in June 2006. The URAT project aims at 5 mas positions for 14 to 18 th mag stars, including proper motions and parallaxes with a limiting magnitude of $R=21$. 